\title{
Adult age differences in acquisition and retention of frequency-of-occurrence information for actions
}

\author{
ZHITANG LIU and DONALD H. KAUSLER \\ University of Missouri, Columbia, Missouri
}

\begin{abstract}
Adult age differences in frequency-of-occurrence memory for actions performed zero, one, or two times in the laboratory were investigated shortly after the actions had been performed and after a retention interval of $24 \mathrm{~h}$. A moderate age difference in accuracy of frequency judgments was found on the immediate retention test. Forgetting over $24 \mathrm{~h}$ was moderate for both young adult and elderly adult subjects. A negligible interaction between age and retention interval suggested comparable forgetting rates for young and elderly subjects, at least over $24 \mathrm{~h}$.
\end{abstract}

A number of studies have reported either the absence of adult age differences or moderate age-related deficits in the accuracy of frequency-of-occurrence memory of words in a lengthy study list (e.g., Freund \& Witte, 1986; Kausler \& Puckett, 1980; see Kausler, 1990, for a review). Unknown, however, is the extent of adult age differences in frequency-of-occurrence memory for another class of episodic events-namely, actions performed in the laboratory (e.g., tapping a foot, rolling dice). One of the objectives of the present study was to test for adult age differences in the accuracy of frequency memory when actions served as the episodic events that varied in their frequencies of occurrence (zero, one, or two). From the perspective of multiple-trace theory (Hintzman \& Block, 1971), subjects are required to discriminate among actions on the basis of the number of their memory traces generated during a performance phase. In this study, such frequency discriminations had to be made among zero, one, and two memory traces. With words as the source of the episodic events, young adults have been found to make frequency discriminations between one and two occurrences with an accuracy that is well above the chance level (e.g., Greene, 1988).

A second objective was to test for the extent of age differences in the forgetting of frequency information over a retention interval of $24 \mathrm{~h}$. Previous studies on age differences in retention and forgetting have typically focused on the forgetting of content, with words serving as the source of content (see Kausler, 1991, for a review). Forgetting, however, extends to the noncontent attributes of episodic events as well as their contents. These attributes

This study was based on part of a thesis by the first author submitted to the Graduate School, University of Missouri-Columbia, in partial fulfillment of the requirements for the Master of Arts degree. The research was supported by Research Grant AG08214 awarded by the National Institute on Aging to the second author. We would like to express our appreciation to Karen Lieberwitz, Judith Wiley, and Roberta Wood for their contributions to the conduct of this study. Correspondence should be addressed to D. H. Kausler, Department of Psychology, University of Missouri, Columbia, MO 65211. include the frequency with which the events occurred and the temporal order in which they occurred. Little is known about age differences in the retention of these attributes. An exception is for the temporal attribute. Kausler and Wiley (1990) reported seemingly comparable rates of forgetting, as indicated by the absence of an age $\times$ retention interval interaction, when actions performed in the laboratory served as the episodic events. Interpretation of their results is complicated, however, by the pronounced age-related deficit found for temporal memory shortly after the actions had been performed. Researchers in retention have long debated the interpretation of an interaction, or the absence of an interaction, when groups being compared for forgetting rates differ in their level of initial acquisition (e.g., Loftus, 1985). For the present study, it was anticipated that the age difference in the initial acquisition of frequency-of-occurrence information would be sufficiently modest to avoid this problem of interpreting whatever age difference in rate of forgetting that might be found.

\section{METHOD}

\section{Design}

The design was a $2 \times 3 \times 2$ mixed factorial. The between-subject variable was age (young, elderly), and the within-subject variables were frequency of occurrence in the performance series (zero, one, or two) and retention interval (immediate or $24 \mathrm{~h}$ ).

\section{Subjects}

The young subjects consisted of 18 undergraduate students (13 women, 5 men) in an introductory psychology course who received course credit for their participation in the study. They ranged in age from 18 to 22 years, with a mean age of 18.94 years $(S D=1.39)$. The elderly subjects consisted of 18 community dwelling residents ( 10 women, 8 men) of Columbia, MO, who were paid for their participation. They ranged in age from 64 to 78 years, with a mean age of 71.83 years $(S D=3.79)$. Mean years of formal education were $13.39(S D=1.20)$ for the young subjects and $16.67(S D=3.24)$ for the elderly subjects $[t(34)=4.03$, $p<.001]$. Each subject rated his or her current health status on a 5point scale $(5=$ poor, $1=$ excellent $)$. Mean health scores were 1.50 $(S D=0.61)$ for the young subjects and $1.56(S D=0.62)$ for the elderly subjects $[t(34)<1]$. After completion of the retention test on the second day, each subject completed the second half of the WAIS-R vocabulary test. Mean scores were $24.06(S D=8.89)$ for the young subjects and $34.83(S D=5.43)$ for the elderly subjects $[t(34)=4.39, p<.001]$. 


\section{Materials}

Sixty actions entered into an initial pool. They were randomly assigned to three sets (A, B and C) of 20 actions each. From these sets, three different lists of action events were formed. For the first list, Set A actions were not performed at all ( 0 frequency), Set B actions were performed once ( 1 frequency), and Set $\mathrm{C}$ actions were performed twice ( 2 frequency). For the other two lists, the sets were rotated in their frequency assignments to assure that each of the 60 actions served equally often in the 0,1 , and 2 frequency-of-occurrence conditions. Six subjects at each age level were assigned randomly to each list.

For each study list, there were two different lists for the frequency judgment tests. One was for the immediate retention test; the other was for the 24-h retention test. Each test list contained verbal statements for 30 actions: 10 of 0 prior frequency, 10 of 1 frequency, and 10 of 2 frequency. For each frequency value, 10 actions were randomly assigned to Set 1 and 10 actions were assigned to Set 2 . For half of the subjects at each age level, Set 1 actions were tested immediately after performance and Set 2 were tested after $24 \mathrm{~h}$. The sets were reversed for the remaining subjects. On each retention test list, there were two different random orders of the actions.

\section{Procedure}

The subjects were informed that they would be performing a number of actions, some once and others twice, and that they would be asked at the end of the series how often each action had been performed (Day 1 retention test). No mention was made of the delayed retention test to be given $24 \mathrm{~h}$ later (Day 2 retention test). They were asked to return the next day to perform on a different task. On Day 2, they were given the retention test prior to receiving the unrelated task. The actions were performed on Day 1 at a rate of $5 \mathrm{sec}$ per single performance of an action, with the experimenter giving a verbal command for each action (e.g., "Roll the dice"). For actions performed twice, there were three to five intervening actions before the repetition.

Shortly after completing the series of actions on Day 1, the subjects received a frequency judgment test for 30 randomly ordered action statements ( 10 each for 0,1 , and 2 frequency) in which there were three columns labeled " 0 ," " 1 ," and " 2 ," They were instructed to check for each action the number of times they believed they had performed it. It was explained to them that some of the actions listed were not performed at all and that these actions should be checked in the 0 frequency column. The retention test on Day 2 was identical to that of Day 1, except, of course, that there was a new list of 30 actions.

\section{RESULTS AND DISCUSSION}

A $2 \times 3 \times 2$ mixed analysis of variance of accuracy scores (i.e., proportion of correct assignments of frequency values of 0,1 , or 2 to actions; see Table 1 for means) yielded significant main effects for age $[F(1,34)$ $\left.=23.32, \omega^{2}=0.07, p<.001\right]$, action frequency $\left[F(2,68)=9.68, \omega^{2}=0.06, p<.001\right]$, and retention

Table 1

Means and Standard Deviations of Accuracy of Frequency Judgment Scores (Proportions of Actions With Frequency of Occurrence Correctly Identified)

\begin{tabular}{|c|c|c|c|c|c|c|}
\hline & \multicolumn{6}{|c|}{ Action Frequency } \\
\hline & \multicolumn{2}{|c|}{0} & \multicolumn{2}{|c|}{1} & \multicolumn{2}{|c|}{2} \\
\hline & Day 1 & Day 2 & Day 1 & Day 2 & Day 1 & Day 2 \\
\hline \multicolumn{7}{|c|}{ Young } \\
\hline$M$ & 0.98 & 0.98 & 0.91 & 0.81 & 0.93 & 0.78 \\
\hline$S D$ & 0.04 & 0.05 & 0.10 & 0.18 & 0.07 & 0.17 \\
\hline \multicolumn{7}{|c|}{ Elderly } \\
\hline$M$ & 0.88 & 0.82 & 0.77 & 0.66 & 0.88 & 0.64 \\
\hline$S D$ & 0.18 & 0.19 & 0.19 & 0.23 & 0.12 & 0.19 \\
\hline
\end{tabular}

interval $\left[F(1,34)=47.00, \omega^{2}=0.16, p<.001\right]$. The action frequency $\times$ retention interval interaction was also significant $\left[F(2,68)=10.80, \omega^{2}=0.07, p<.001\right]$. Most importantly, none of the interactions involving age approached significance $(F \mathrm{~s}<1)$. The age effect resulted from the fact that the overall mean score (pooled for action frequency and retention interval) was higher for the young subjects $(0.90)$ than for the elderly subjects $(0.78)$. The action frequency effect (pooled for age and retention interval) resulted from the higher score for 0 frequency actions $(0.92)$ than for either 1 frequency or 2 frequency actions ( 0.79 and 0.81 , respectively). The retention interval effect resulted from the higher mean score (pooled for age and action frequency) on the immediate retention test $(0.89)$ than on the $24-h$ retention test $(0.78)$. The item frequency and retention interval effects, however, need to be interpreted in light of the significant action frequency $X$ retention interval interaction effect. From Table 1 it may be seen that the interaction reflects the greater forgetting from Day 1 to Day 2, at each age level, of 1 and 2 frequency actions than of 0 frequency actions.

As may be seen in Table 1, there was a moderate agerelated deficit in accuracy of frequency judgments on Day 1 for each level of frequency of occurrence. Pooled over the three levels, the young subjects had a mean accuracy score of 0.94 and the elderly subjects had a mean score of 0.84 . Frequency-of-occurrence memory for actions appears to be moderately age sensitive, and to about the same extent as frequency-of-occurrence memory for words. The relative age difference was $10.6 \%$, an agerelated decline that approximates that found when words serve as the source of episodic events (see Kausler, 1990). The magnitude of the present age-related deficit is considerably less than that found for temporal memory when either continuous activities (Salthouse, Kausler, \& Saults, 1988 ) or discrete actions (Kausler \& Wiley, 1990) serve as the episodic events. The absence of an age $\times$ retention interval interaction in the present study suggests that the rate of forgetting frequency information was comparable for the young and elderly subjects. However, the presence of a moderate age difference in initial acquisition, as measured by Day 1 scores, again forces a cautious interpretation of the negligible interaction effect: Nevertheless, what is clearly apparent from Table 1 is that both the young and the elderly subjects displayed only a moderate degree of forgetting over $24 \mathrm{~h}$. For the young subjects, the overall mean accuracy score declined from 0.94 on Day 1 to 0.86 on Day 2; for the elderly subjects, the decline was from 0.84 on Day 1 to 0.71 on Day 2. At each age level, the magnitude of the decline is much less than that found by Kausler and Wiley (1990) for the temporal memory of a series of actions.

\section{REFERENCES}

FreUND, J. S., \& WITTE, K. L. (1986). Recognition and frequency judgments in young and elderly subjects. American Journal of Psychology, 99, 81-102.

GREENE, R. L. (1988). Generation effects in frequency judgment. Jour- 
nal of Experimental Psychology: Learning, Memory, \& Cognition, 14, 298-304.

Hintzman, D. L., \& Block, R. A. (1971). Repetition and memory: Evidence for a multiple-trace hypothesis. Journal of Experimental Psychology, 88, 297-306.

KAUSLER, D. H. (1990). Automaticity of encoding and episodic memory processes. In E. A. Lovelace (Ed.), Aging and cognition: Mental processes, self awareness and interventions (pp. 29-67). Amsterdam: North-Holland.

KAUSLER, D. H. (1991). Experimental psychology, cognition, and human aging. New York: Springer-Verlag.

Kausler, D. H., \& PUCKetT, J. M. (1980). Frequency judgments and correlated cognitive abilities in young and elderly adults. Journal of Gerontology, 35, 376-382.

KaUSler, D. H., \& Wiley, J. (1990). Temporal memory and content memory for actions: Adult age differences in acquisition and retention. Experimental Aging Research, 16, 147-150.

LoFTUs, G. R. (1985). Evaluating forgetting curves. Journal of Experimental Psychology: Learning, Memory, \& Cognition, 11, 397-406. Salthouse, T. A., Kausler, D. H., \& Saults, J. S. (1988). Investigation of student status, background variables, and feasibility of standard tasks in cognitive aging research. Psychology \& Aging, 3, 29-37.

(Manuscript received September 4, 1992.)

\title{
News and Notes from NSF
}

Most proposals to the National Science Foundation for research and related activities in animal learning and behavior are reviewed by the Advisory Panel for Animal Learning and Behavior. Current members of the Panel are Peter D. Balsam (Barnard College), Carol M. Berman (SUNY, Buffalo), Michael D. Breed (University of Colorado), Ruth E. Buskirk (University of Texas), Edmund J. Fantino (University of California, San Diego), Stewart H. Hulse (Johns Hopkins University), Alan C. Kamil (University of Nebraska), Patricia L. Schwagmeyer (University of Oklahoma), Judy A. Stamps (University of California, Davis), George W. Uetz (University of Cincinnati), and Ken Yasukawa (Beloit College).

The Advisory Panel for Animal Learning and Behavior wishes to maintain the highest methodological standards in observational as well as experimental research. Proposals for observational studies of behavior should describe how observers are trained, how interobserver reliability is assessed, and how observer bias is prevented or controlled.

Most proposals with major neurobiological aspects are reviewed by the following Neuroscience Programs. Each Program Director's name appears in parentheses:

\author{
Cognitive, Computational, and Theoretical Neurobiology \\ (Donald H. Edwards) \\ Neural Mechanisms of Behavior (Christiana M. Leonard) \\ Sensory Systems (Christopher J. Platt) \\ Neuroendocrinology (Kathie L. Olsen) \\ Developmental Neuroscience (Steven C. McLoon) \\ Neuronal and Glial Mechanisms (Sanya A. Springfield)
}

As a result of the recent reorganization of Biological, Behavioral, and Social Sciences at NSF, all of the Programs named above are in the Division of Integrative Biology and Neuroscience (IBN), one of four Divisions in the Directorate for Biological Sciences (BIO).

Target dates for submission of proposals to be reviewed by the Advisory Panel for Animal Learning and Behavior have changed. The new target dates are June 15 and December 15 each year. Neuroscience target dates remain January 15 and July 15. Proposals for Minority Research Initiation and Research in Undergraduate Institutions are reviewed by the disciplinary panels on the same schedule as other research proposals.

Such competitions as NSF Graduate Fellowships, Presidential Faculty Fellows, NSF Young Investigators, Research Planning Grants and Career Advancement Awards for Women Scientists, and the Special Competition for Basic Research in Conservation and Restoration Biology have specially.convened panels and annual deadlines for proposal submission.

For more information, call Fred Stollnitz, Program Director for Animal Behavior, at 202-357-7949, or any of the Neuroscience Program Directors at 202-357-7040. The IBN Fax number is 202-357-7846. 